\title{
SPACE TIME MANIFOLDS AND CONTACT STRUCTURES
}

\author{
K.L. DUGGAL \\ Department of Mathematics and Statistics \\ University of Windsor, Windsor \\ Ontario, Canada N9B 3P4 \\ (Received February 27, 1989)
}

\begin{abstract}
A new class of contact manifolds (carring a global non-vanishing timelike vector field) is introduced to establish a relation between spacetime manifolds and contact structures. We show that odd dimensional strongly causal (in particular, globally hyperbolic) spacetimes can carry a regular contact structure. As examples, we present a causal spacetime with a non regular contact structure and a physical model [Göde1 Universe] of Homogeneous contact manifold. Final1y, we construct a model of 4-dimensional spacetime of general relativity as a contact CR-submanifold.
\end{abstract}

KEY WORDS AND PHRASES. Contact manifold, global timelike vector field, spacetime manifold, Lorentzian geometry, submanifold.

1980 AMS SUBJECT CLASSIFICATION CODES. 53C25, 83C50.

1. INTRODUCTION.

A smooth $(2 n+1)$-dimensional manifold $N$ is called contact manifold if it carries a global 1-form $n$ such that

$$
n \Lambda[d n)^{n} \neq 0
$$

everywhere on $N$, where $d \eta$ is the exterior derivative of the contact from $\eta$. $N$ is then orientable. The name contact is due to S. Lie [1]. Classically there have been two large classes of contact manifolds, namely, the principle circle bundle of the boothby-wang fibrations including the odd-dimensional spheres and the tangent sphere bundles. A thorough disussion may be found in [2]. The theory of contact manifolds has been widely used in mathematical physics. For example, in thermodynamics [3], a contact manifold is named as thermodynamic phase space (TPS) with contact form $n=$ du - Tds + pdv +..., where $u, s, v, p$ and $T$ are the internal energy, the entropy, the volume, the pressure and the temperature respective1y.

Contact manifolds have an interplay with Cauchy Riemann (CR) manifolds [4,5] in the following way. The 2 -dimensional contact distribution $D=\{X \varepsilon T N / n(X)=0\}$ has a complex structure. Thus, the complexification of $D$ has a holomorphic sub-bundle $H$ such that $(\mathrm{N}, \mathrm{H})$ is a CR-manifold if the contact structure is normal [6]. Recent1y, the present author introduced a new area of research, namely, Lorentzian Geometry of CR submaniflds with applications to relativity [7,8]; cf. also [9]. As normal contact manifold is an example of CR manifolds (see Blair [2] p. 62), a systematic study on the Lorentzan geometry (mathematical theory for relativity) of contact manifolds is needed. 
Motivated by above, as a first step, the objective of this paper is to establish a relation between spacetime manifolds and contact structures.

Our study is in line with the latest trend of relating the Riemannian and Lorentzian geometry (see, for example [13, 14, 17]).

\section{PRELIMINARIES.}

A spacetime $(M, g)$ is a connected smooth Hausdorff manifold of dimension $>2$ with a Lorentz metric $g$ of signature $(-,+, \ldots,+)$ and is time oriented, that is, has a global timelike vector field. All non-compact manifolds admit Lorentz metrics, whereas, a compact manifold is Lorentz iff its Euler characteristic vanishes. Any compact spacetime contains a closed timelike curve. For example, on the cylinder $R \times S^{1}$ with $d s^{2}=-d t^{2}+d \theta^{2}$, the circles $t=$ const. are closed timelike curves. In relativity, each point of spacetime corresponds to an event relative to time. Thus, physically, closed timelike curves are forbidden as this raises the possibility that a person might meet himself in the past. Therefore, all physical spacetimes are assumed non-compact. Let $\mathrm{C}:[\mathrm{a}, \mathrm{b}] \rightarrow M$ be a curve in $M$. The point $p \varepsilon M$ is called the end point of $C$ for $t=b$ if $\lim C(t)=p$. A nonspacelike curve is future (resp., past) $t \rightarrow b-$

inextendible if it has no future (resp., past) endpoint. A nonspacelike future (resp. past) inextendible curve $C$ does one of the following:

(1) Traps within a compact set $\&$.

(2) Does not trap in any compact set but continually re-enter a compact set $\beta$.

(3) Does not trap in any compact set and does not re-enter in any such set more than a finite number of times.

If (3) holds then $C$ goes off to the edge of spacetime to infinity or a singularity point. For (1) and (2), $C$ is called totally and partially imprisoned in $\&$, respective1y. Carter [10] has given following example of a causal spacetime $M$ having imprisoned nonspacelike inextendible curves. The metric is:

$$
d s^{2}=(\cosh t-1)^{2}\left(d y^{2}-d t^{2}\right)-d t d y+d z^{2}
$$
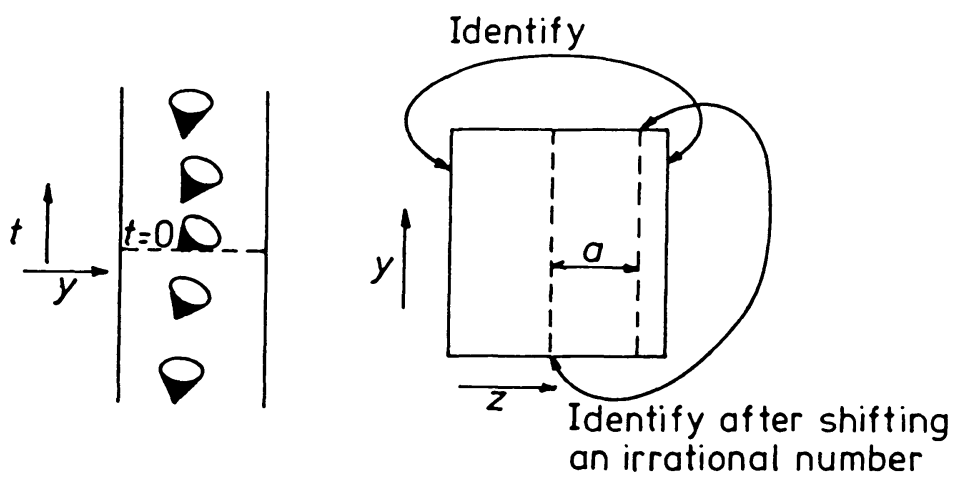

(Fig. 1)

$M=R \times S^{1} \times S^{1}=\left\{(t, y, z) \varepsilon R^{3}:(t, y, z) \sim(t, y, z+1)\right.$ and $(t, y, z) \sim(y, y+1, z+a)$, where a is an irrational number. A Cauchy hypersurface $S$ in $M$ is a subset that meets exactly once every inextendible nonspacelike curve in $M . \quad M$ is globally hyperbolic iff it 
admits a Cauchy hypersurface. According to Geroch [11] a globally hyperbolic spacetime is a product manifold of the form $\left(M=R \times S: g=-d t^{2} G\right)$ with (S,G) a compact Riemannian manifold. Minkowski spacetime and Einstein static universe are simple examples. Given any two points $p, q$ of $M, q$ is in the chronological future (resp. past) of $p$, denoted by $p \ll q$ (resp. $q \ll p$ ), if there is a future (resp. past) directed timelike curve from $p$ to $q$. The chronological future (resp. past) of $p$ are the sets $I^{+}(p)=\{q \in M: p \ll q\}$ and $I^{-}(p)=\{q \varepsilon M: q \ll p\}$. The causal future (resp. past) of $p$ are the sets $J^{+}(p)=\{q \varepsilon M: p<q\}$ and $J^{-}(p)=\{q \varepsilon M: p<q\}$ for nonspacelike curves. This means that $M$, with no closed nonspacelike curve is causal space. Also, as any compact spacetime contains a closed timelike curve, it fails to be causal. The sets $I^{+}(p)$ and $I^{-}(p)$ are always open in any space time, but the sets $\mathrm{J}^{+}(\mathrm{p})$ and $\mathrm{J}^{-}(\mathrm{p})$ are neither open nor closed in general. $M$ is strongly causal if its every point has arbitrarily small neighbourhoods which no nonspacelike curve intersects more than once. A strongly causal $M$ is globally hyperbolic, tf for each p, $q$ of $\mathrm{M}, \mathrm{J}^{+}(\mathrm{p}) \cap \mathrm{J}^{-}(\mathrm{q})$ is compact.

PROPOSITION 1. If the strong causality condition holds on a compact set of a spacetime, then there can be non imprisoned inextendible curves (Hawking and E11is [12]). For details on above (with examples) we refer [13].

\section{CONTACT SPACE TIMES.}

A (2n+1)-dimensional smooth manifold $N$ is called an almost contact metric manifold if there exists a tensor field $\phi$ of type $(1,1)$, a vector field $\xi$, 1form $n$ and a semi-Riemannian metric $g$ such that:

$$
\begin{aligned}
& \phi^{2}=-I+n \otimes \xi, \phi \xi=0, n \phi=0, n(\xi)=1 \\
& g(\xi, \xi)=\varepsilon, g(\phi X, \phi Y)=g(X, Y)-\varepsilon n(X) n(Y)
\end{aligned}
$$

where $\varepsilon=+1$ or -1 according as $\xi$ is spacelike or timelike and $\operatorname{rank}(\phi)=2 n$. If $d n(X, Y)=g(\phi X, Y)$ for every $X, Y$ of $N$, then $N$ is called a contact metric manifold. For $\varepsilon=+1$ and $g$ definite, $N$ is the usual contact metric structure [2]. Here, we study a larger class to allow indefinite metric. For example, if $\varepsilon=-1$, then $N$ contains a timelike vector field. However, the signature of $g$ is restricted by the following result.

PROPOSITION 2. The 2n-dimensional contact distribution $D(n=0)$ of a contact manifold $(n>2)$ cannot carry a Lorentz metric.

PROOF. D admits a Hermitian structure which cannot carry a Lorentz metric unless $\mathrm{n}=1$ (for detalls see Flaherty [14]). Based on above proposition the following holds. THEOREM 1. For a contact metric manifold $\mathrm{N}$, the following are equivalent:

(a) $\mathrm{N}$ is a spacetime manifold

(b) the contact vector field $\xi$ is timelike and the contact distribution $D$ (defined by $n=0$ ) is spacelike lexcept when $\operatorname{dim}(N)=3$, then $\xi$ spacelike and $D$ timelike is possible].

In this paper, a contact manifold with a Lorentz metric will be called a contact spacetime. An almost contact manifold is said to be normal if the Nijenhuis tensor of $\phi$ satisfies:

$$
[\phi, \phi]+2 d n \xi \xi=0
$$


EXAMPLE. Consider a (2n+l)-dimensional spacetime manifold $\mathrm{N}$ with a local coordinate system $\left(x^{i} ; y^{1}, t\right) i=1, \ldots, n$. N being time ${ }_{n}$ oriented admits a global timelike vector field, say $\xi$. Define a 1 -form $n=1 / 2\left(d t-\sum_{1} y^{1} d x^{1}\right)$ so that

$\xi=2 \partial_{t}$. Then, from a classical theorem of Darboux [2], $N$ is a contact manifold with contact from $n$ and timelike contact vector field $\xi$. The Lorentz metric $g$ :

$$
g=1 / 4\left[\sum_{1}^{n}\left(\left(d x^{1}\right)^{2}+\left(d y^{i}\right)^{2}\right)-n \otimes n\right]
$$

gives a contact metric structure to the spacetime $N$ for $\varepsilon=-1$. Therefore, $N$ is a contact spacetime. With respect to an orthonormal $\phi$-basis $\left(U_{1} ; U_{n+1}, \xi\right)$, where

$$
U_{1}=2 \partial_{y} 1, U_{n}+1=2\left(\partial_{x} i+y^{1} \partial_{t}\right), \phi U_{1}=U_{n+1}, \phi U_{n+1}=-U_{1}
$$

the metrix of the components of $g$ is given by:

$$
\frac{1}{4}\left[\begin{array}{ccc}
\delta_{1 j}-y^{1} j & 0 & y^{1} \\
0 & \delta_{i j} & 0 \\
-y^{j} & 0 & -1
\end{array}\right]
$$

THEOREM 2. An odd dimensional globally hyperbolic spacetime can carry a contact structure.

PROOF. Consider an almost Hermitian manifold $\left[M^{2 n}, G, J^{2}=-1\right]$ and $G(J X, J Y)=$ $G(X, Y)$ for every $X, Y$ of $M^{2 n}$. Construct a globally hyperbolic spacetime $N=\left\{R \times M^{2 n}, g=-d t^{2}+G\right\}$. Denote a vector field on $N$ by $\bar{X}=\left(n(\bar{X}) \frac{d}{d t}, x\right)$ where $X$ is tangent to $M^{2 n}, t$ is the coordinate of $R$ and $n(\bar{X})$ is a smooth function on $N$. Set $n=d t$ so that $\xi=\left(\frac{d}{d t}, 0\right)$ is timelike global vector field. Then with

$$
\begin{gathered}
\phi\left(n(\bar{X}) \frac{d}{d t}, X\right)=(0, J X) \\
g\left[\left(n(\bar{X}) \frac{d}{d t}, X\right), \quad\left(n(\bar{Y}) \frac{d}{d t}, Y\right)\right]=G(X, Y)-n(\bar{X}) n(\bar{Y}),
\end{gathered}
$$

we recover a contact metric structure on $\mathrm{N}$ for $\varepsilon=-1$.

In particular, let $\mathrm{R}_{\mathrm{q}}^{\mathrm{n}}$ be $\mathrm{n}$-dimensional pseudo-Euclidean space of signature ($, \ldots,-,+, \ldots,+)$ with $q$ negative and $n-q$ positive elgenvalues. Hence, for a local coordinate system $\left(x_{1}\right), 1=1, \ldots, n$, its metric is given by:

$$
d s^{2}=-\sum_{1}^{q} d x_{1}^{2}+\sum_{q+1}^{n} d x_{1}^{2}
$$

Define for $r>0$ (see Wolf [15 section 4.2])

$$
s_{1}^{n}=\left\{X \in R_{1}^{n+1}:-x_{1}^{2}+x_{2}^{2}+\ldots+x_{n+1}^{2}=r^{2}\right\}
$$

and

$$
H_{1}^{n}=\left\{X \& R_{1}^{n+1}:-X_{1}^{2}-x_{1}^{2}+\ldots+x_{n+1}^{2}=-r^{2}\right\}
$$


Topologically, $S_{1}^{n}$ is $R^{1} \times S^{n-1}$ and $H_{1}^{n}$ is $S^{1} \times R^{n-1}$. Also, $S_{1}^{n}$ is a Lorentzian analogue of the sphere of radius $r$ with positive curvature $r^{-2}$. The Universal covering $\tilde{H}_{1}^{n}$ of $H_{1}^{n}$ is topologically $R_{1}^{n}$, and, thus, a hyperbolic space of negative curvature $-r^{-2}$. $\quad S_{1}^{n}\left(\tilde{H}_{1}^{n}\right)$ are called de-Sitter (anti de-sitter) spaces [12]. $s_{1}^{n}$ is globally hyperbolic but $\widetilde{\mathrm{H}}_{1}^{\mathrm{n}}$ is not. Thus, we have

COROLLARY. Odd dimensional de-sitter spaces can carry a contact structure.

\section{REGULAR CONTACT SPACE TIMES.}

A contact structure on $N$ is regular if every point $p$ of $N$ has a cubical coordinate neighbourhood $\mu$ such that the integral curves of $\xi$ passing through $\mu$ pass this neighborhood only once. If $N$ is compact, then the maximal integral curves of $\xi$ are homeomorphic to circles and therefore Boothby-Wang theorem [16] will hold. For noncompact spacetimes, comparing the definitions of strongly causal spacetimes and regular contact spaces, we get

THEOREM 2. An odd dimensional strongly causal spacetime can carry a regular contact structure.

COROLLARY. Odd dimensional globally hyperbolic spacetimes can carry a regular contact structure.

Well-known examples are Minkowski spacetime, Lorentz spheres and Robertson-Walker spacetimes.

On the other hand, there exist causal spacetimes which can not carry a regular contact structure. To illustrate this point consider a 3-dimensional contact manifold $M_{3}$ defined by $n=\cosh z d t-\sinh z$ dy and $\xi=\cosh z \partial_{t}+\sinh z \partial y$ for a coordinate system $(t, y, z)$. The Lorentz metric

$$
\begin{aligned}
g & =\cosh 2 z d y^{2}+d z^{2}-(1+\sinh 2 z) d t d y-n \quad \eta \\
& =\cosh ^{2} z\left[d y^{2}-d t^{2}\right]+d z^{2}-d t d y .
\end{aligned}
$$

gives a contact metric structure on spacetime $M_{3}$. In particular, for $\left.\cosh ^{2} z=\operatorname{cosht}^{-1}\right)^{-1}$ and $t>0, M_{3}$ is Carter's example (see section 2) of a causal spacetime with Inextendible nonspacelike imprisoned curves. Note that the integral curves of $\xi$ through $(y, y+1, a)$ Induce an irrational flow as a is 1rrational. Therefore $\xi$ is not regular.

The discussion, so far, opens the way to address the following basic problem.

\section{"Characterise regular contact spacetimes"}

For the characterisation of regular contact Riemannian manifolds, we refer [16]. The following results may be useful.

PROPOSITION 3 (0'Neill [17]). Maximal integral curves of a vector field $V$ of a spacetime are inextendible.

This means that the maximal integral curves of the regular contact vector field $\xi$ are extendible. On the topology of such spacetimes, we know that Open sets of the form $\mathrm{I}^{+}(p) \cap \mathrm{I}^{-}(q)$ for any $p, q$ of any spacetime $\mathrm{N}$ form a basis of a topological structure on N, called Alexandrov topology. This topology is related with the strongly causal spacetimes (and, therefore, regular contact spacetimes) by the 
PROPOSITION 4. (Penrose [18]) The Alexandrov topology for a spacetime $N$ agrees with the given manifold topology iff $\mathrm{N}$ is strongly causal.

A contact manifold $\mathrm{N}$ is said to be homogeneous if there is a connected Lie group $G$ acting transitively and effective as a group of differentiable homomorphism on $N$ which leave $n$ Invariant. Boothby and Wang [16] have proved that the integral curves of $\xi$ are necessarily regular for homogeneous contact manifolds but $N$ need not be compact. Also, following holds:

THEOREM 4 (Boothby-Wang) [16]). The integral curves of the contact vector field $\xi$ of a homogeneous contact manifold are either closed curves or open arcs.

Relating above result with homogeneous spacetimes, we present the following example:

Kurt Gơdell [19] discovered a homogeneous spacetime, called Gödell Universe, whose metric $\mathrm{g}$ is a direct sum of the metric:

$$
g_{1}=-d t^{2}+d x^{2}-1 / 2 \exp (2(\overline{\sqrt{2}}) a x) d y^{2}-2 \exp ((\sqrt{2}) a x) d t d y,
$$

on the 3-dimensional manifold $M_{3}$ defined by the coordinates $(t, x, y)$, where $a>0$ is a constant, and the metric $g_{2}=\mathrm{dz}^{2}$ on the manifold $\mathrm{R}^{l}$ defined by the coordinate $z$. For solutions of the Einstein's field equations, it is sufficient to consider only $\left(M_{3}, g_{1}\right)$. Transforming into new coordinates $\left(t^{\prime}, r, \phi\right)$ by

$$
\begin{aligned}
& \exp ((\overline{\sqrt{2}}) a x)=\cosh 2 r+\cos \phi \sinh 2 r, \\
& \text { ay } \exp ((\overline{\sqrt{2}}) a x)=\sin \phi \sinh 2 r, \\
& \tan 1 / 2\left(\phi+a t-(\overline{\sqrt{2}}) t^{\prime}\right)=\exp (-2 r) \tan 1 / 2 \phi,
\end{aligned}
$$

the metric $g_{1}$ takes the new form $g_{1}=2 a^{-2}\left[-d t^{2}+d r^{2}-\left(\sinh ^{4} r-\sinh ^{2} r\right) d \phi^{2}+2(\sqrt{2}) \sinh ^{2} r d \phi d t\right]$.

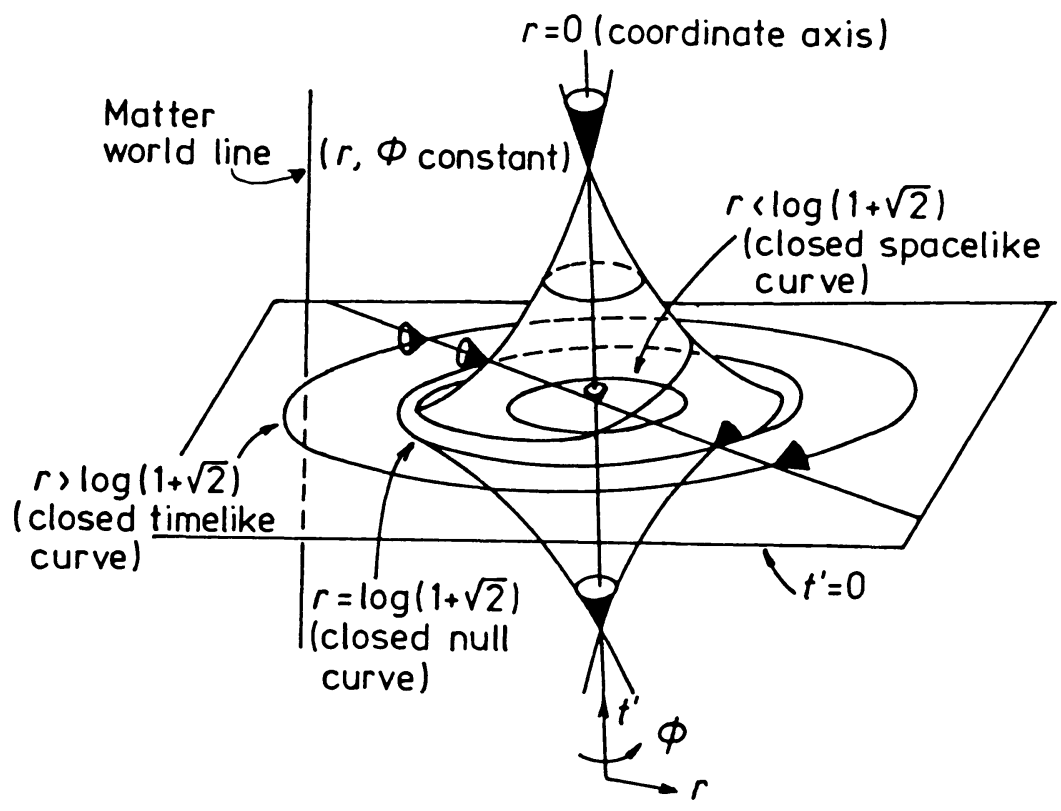


The flow vector of the fluid is $u=(a / \sqrt{2}) \partial_{t} \cdot$ The space is rotationally symmetric about the axis $r=0$ (see fig. 2). The 1ight cones on the axis $r=0$ contain the direction of $u$. The light cone opens out and tips over as $r$ increases resulting in closed timelike curves. At a radius $r=10 g(1+\overline{\sqrt{2}}), \partial \phi$ is a nul1 vector. For a greater value of $r, \partial_{\phi}$ is a timelike vector and circles of constant $r$, $t^{\prime}$ are closed timelike curves. $\left(M_{3}, g_{1}\right)$ is a contact spacetime define by

$n=(\overline{\sqrt{2}} / a)\left(d t^{\prime}+A d \phi\right), \xi=(a / \overline{\sqrt{2}})\left[\partial_{t}^{\prime}+A^{-1} \partial_{\phi}\right]$, where $A^{2}=\sinh ^{4} r-s i n h^{2} r$. Thus, Gơdel universe is an example of a homogeneous contact spacetime such that the integral curves of $\xi$ [for $r>\log (1+\sqrt{2})$ and $t^{\prime}$ constant] are closed timelike curves.

Further research in this direction is needed to find the topology of homogeneous contact spacetimes. For results on these topics with respet to Riemanian metric see $[16]$.

5. CONTACT STRUCTURE AND PHYSICAL SPACE TIME.

By a physical spacetime we mean 4-dimensional spacetime in the context of relativity (special or general). As contact manifolds are odd dimensional (there do exist even dimensional contact manifolds [20] which we are not discussing here), to relate them with the physical spacetime, we embed physical spacetime as submanifold of contact manifold. There are three popular classes of submanifolds of contact manifolds. First, invariant submanifolds [21] which inherit almost all properties of the ambient manifold. Second, anti-invariant submanifolds [22]. Third, contact CR manifolds $[4,5]$ (See Goienner [23] for details on the isometric embedding of spacetimes. In particular, every 4-dimensional spacetime $M$ can be seen (locally) as a submanifold of a Minkowski space $R_{1}^{n}$ of $n<10$. Globally, $n<46$ or 87 according as $M$ is compact or noncompact). An invariant submanifold of a contact manifold inherits contact struture and, therefore, mat be ruled out for our purpose. Out of the other two classes, we consider CR submanifolds as they include anti-invariant class.

Let $M$ be a real m-dimensional submanifold of a contact metric manifold

$(\mathrm{N}, \phi, \xi, \eta, g)$ with semi-Riemannian metric metric $g$. We use same symbol $g$ for the metric of $M$. Let $\xi$ be tangent to $M$.

DEFINITION. $M$ is called a contact $C R$ submanifold of $N$ if there exist two distributions $D$ and $D^{\perp}$ on $M$ satisfying

(1) $T M=D \oplus D^{\perp} \oplus\{\xi\}$, where $D, D^{\perp}$ and $\{\xi\}$ are mutually orthogonal to each other;

(2) $D$ is invariant by $\phi[\phi(D)=D]$;

(3) $D^{\perp}$ is anti-invariant by $\phi\left[\phi\left(D^{\perp} \subset T M^{\perp}\right]\right.$

The same concept was studied under the name semi-invariant submanifolds [5]. Here we define a larger class to include indefinite metric. $\operatorname{Dim}(D)=0$ (resp. $\operatorname{dim}\left(D^{\perp}\right)=0$ implies $M$ is anti-invariant (resp. invariant), otherwise it is a non-trivial contact CR submanifold.

Based on above definition, we now construct a mathematical model of 4-dimensional spacetimes as contact CR submanifolds.

MODEL. Let $(\mathrm{N}, \mathrm{g})$ be a 5-dimensional spacetime with a local coordinate system $(x, s, y, z, t)$. Define a 1 -form $\eta=1 / 2[d t-y d x-z d s]$ so that there exists a vector field $\xi=2 \partial_{t}$ on $N$. $N$ has a contact metric structure $(\phi, \xi, n, g)$ with contact form $n$ and

$$
g=1 / 4\left[d x^{2}+d y^{2}+d s^{2}+d z^{2}-n \cdot n\right]
$$


as the defining equations (2) hold for $\varepsilon=-1$. There exists an orthonormal $\phi$-basis $\left(U_{i} ; v_{1} ; \xi\right)$ where

$$
\begin{gathered}
U_{1}=2 \partial_{y}, U_{2}=2 \partial_{z}, v_{1}=2\left(\partial_{x}+y \partial_{t}\right), v_{2}=2\left(\partial_{s}+z \partial_{t}\right), \\
\phi U_{1}=v_{1}, \phi V_{1}=-U_{i}, i=1,2
\end{gathered}
$$

Let $(M, g)$ be a 4-dimensional spacetime embedded in $N$. So that $\left(U_{1}, U_{2}, V_{1}, \xi\right)$ is an orthonormal basis for $T_{p}(M)$. Then, the complexified tangent space CT(M) has a holomorphic subbundle $H$ generated by $(Z, \bar{Z})$ with $Z=U_{1}+i V_{1}$ and $J Z=-1 Z(J$ is complex structure on $H)$. Thus, there exists a real distribution $D=\operatorname{Re}(H+\bar{H})$ generated by $\left(U_{1}, V_{1}\right)$ invariant by $\phi$ and anti-invariant distribution $D^{\perp}=\left\{U_{2}\right\}, D, D^{\perp}$ and $\xi$ are mutually orthogonal to each other. Therefore, by definition, $M$ is a contact $C R$ submanifold of $N$ with timelike contact vector field $\xi$ and spacelike invariant distribution $D$. In general, the dimension of the embedding manifold $N$ may be higher than 5 (subject to restrictions as stated in (23]).

REMARK. It is worth mentioning that the embedding manifold has been used as auxiliary space for deriving physical properties of the embedded spacetime. In particular, several exact solutions of the Einstein's field equations have been found by this technique, at least for some cases of low embedding class (the minimum number of extra dimension is called the embedding class). For example, the maximal analytic extension of the Schwarzschild solution was found by embedding technique [24].

However, unfortunately, no systematic research has been done to find solutions of embedding class greater than two. Difficulty is the lack of effective method for solving embedding equations.

Hopefully, by supplying some differential geometric structure (such as the contact structure discussed in this paper), it may help to provide more insight on the geometry of spacetime needed for physical problems (including finding exact solutions) in relativity.

In another direction, the disussion in this section leads to a new area of research, namely, Lorentzian geometry of CR submanifolds (including contact CR submanifolds as subcase), introduced by the present author $[7,8]$. For related references on some progress in this direction and, in general, bridging the gap between Differential Geometry and Mathematical Physics of Relativity, see [13, 14, 17, 25-27].

\section{ACKNOWLEDGEMENT .}

This research was supported by the Natural Sciences and Engineering Research Council of Canada. 
REFERENCES

1. LIE, S., Theorie der Transformationgruppen, Vo1. 2, Leipzig, Tenbner, 1890.

2. BLAIR, D.E., Contact manifolds in Riemannian geometry, Lecture notes in Math., Springer - Verlag, No. 509, 1976.

3. MRUGALA, R., Submanifolds in the thermodynamic phase space, Rep. Math. Phys. 21 (1985), 197-203.

4. YANO, K. and KON, M., CR Submanifolds of Kahlerian and Sazakian Manifolds, Birkhauser, Boston, 1983.

5. BEJANCU, A., Geometry of CR Submanifolds, Reidel Publishing Company, Holland, 1986.

6. IANUS, S., Sulle varieta di Cauchy - Riemann, Rend. dell Acacademia di Scienze Fisiche and Matemtiche, Napoli, XXXIX (1972), 191-195.

7. DUGGAL, K.L., CR-Structures and Lorentzian geometry, Acta Applicandae Mathematicae $7(1986), 211-223$.

8. DUGGAL, K.L., Lorentzian geometry of CR submanifolds, Acta Applicandae Mathematicae (In Print).

9. PENROSE, R., Physica1 spacetime and non-realizable CR-structures, Am. Math. Soc. 8 (1983), 427-448.

10. CARTER, B., Causal structure in spacetime, Gen. Re1. Gravit. 1 (1970a), 349-391.

11. GEROCH, R.P., Domain of dependence, J. Math. Phys. 11 (1970), 437-449.

12. HAWKING, S.W. and ELLIS, G.F.R., The large scale structure of spacetime, Cambridge Univ. Press, Cambridge, 1973.

13. BEEM, J.K. and EHRLICH, P.E., Global Lorentzian geometry, Marce1 Dekker, New York, 1981.

14. FLAHERTY, E.J., Hermitian and Kahlerian geometry in relativity, Lecture notes in Phys., Springer-verlag, Berlin, No. 46, 1976. 


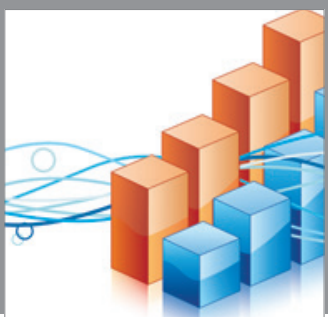

Advances in

Operations Research

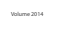

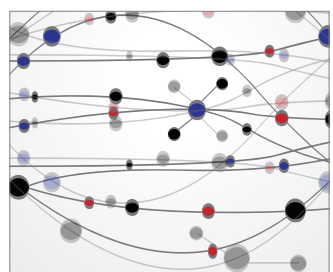

\section{The Scientific} World Journal
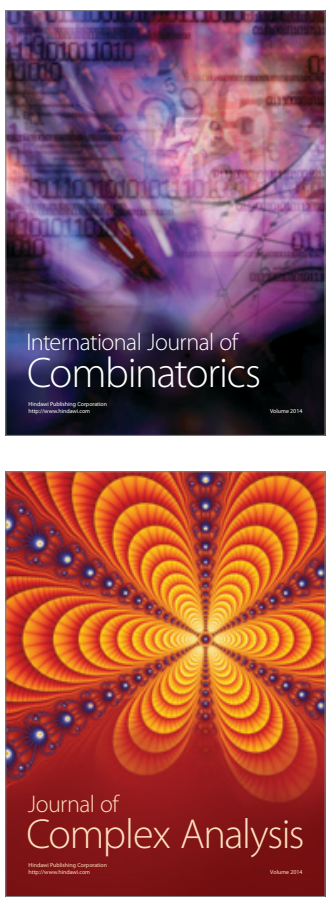

International Journal of

Mathematics and

Mathematical

Sciences
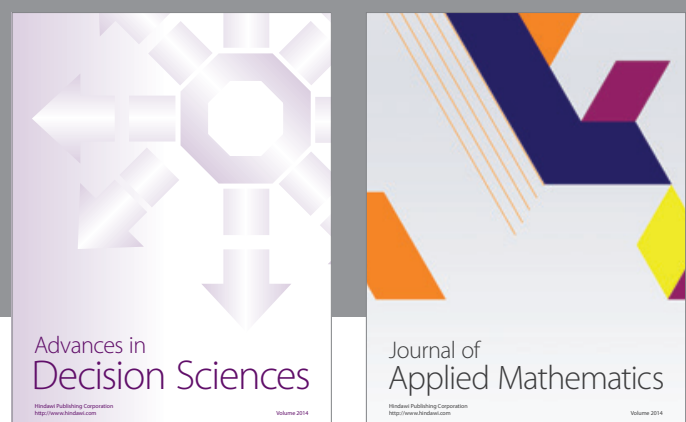

Journal of

Applied Mathematics
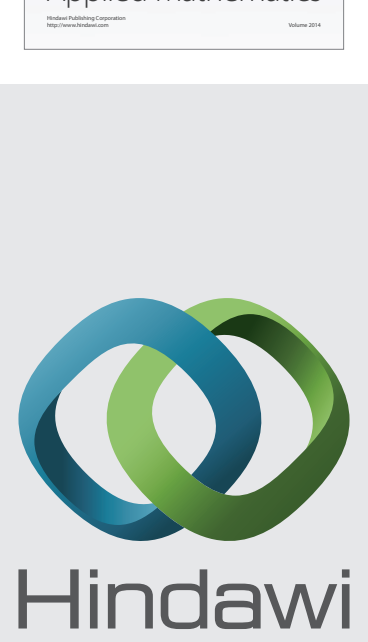

Submit your manuscripts at http://www.hindawi.com
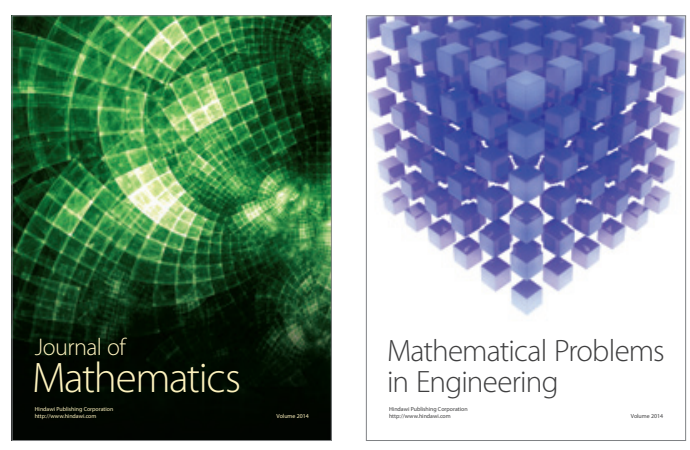

Mathematical Problems in Engineering
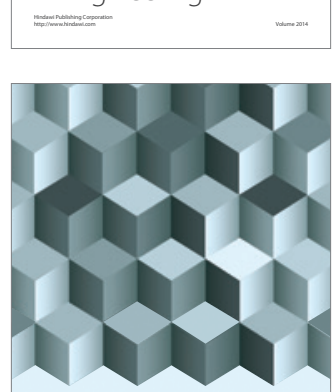

Journal of

Function Spaces
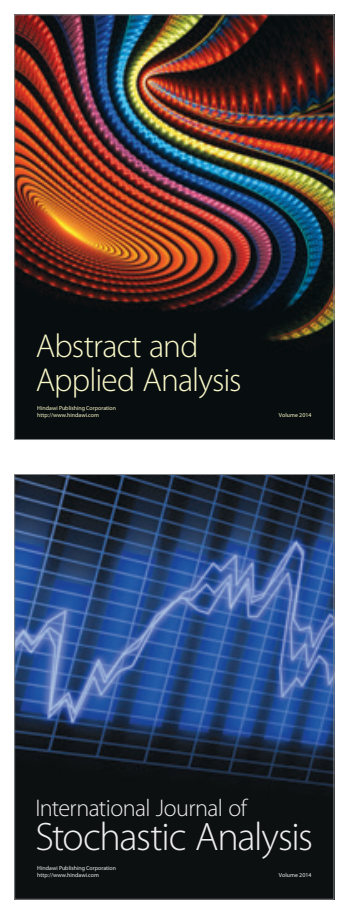

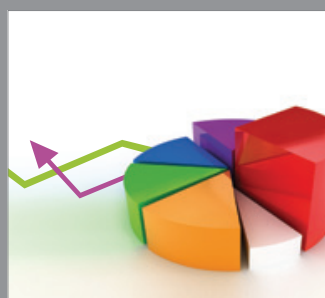

ournal of

Probability and Statistics

Promensencen
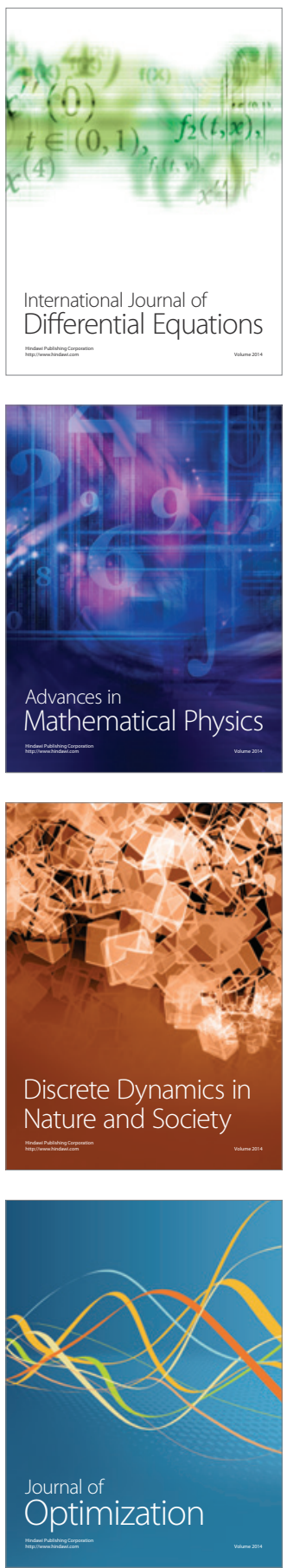